\title{
RECUPERAÇÃO DE MARCAÇÕES ADULTERADAS EM CHAPAS METÁLICAS PELO MÉTODO NÃO DESTRUTIVO DE VISUALIZAÇÃO MAGNETO-ÓPTICA *
}

\author{
Mariana Gaudencio Barbosa Lima ${ }^{1}$ \\ Clara Johanna Pacheco ${ }^{2}$ \\ Maria Cecília de Souza Nóbrega ${ }^{3}$ \\ Gabriela Ribeiro Pereira ${ }^{4}$
}

\section{Resumo}

O presente trabalho se baseia no estudo da recuperação de marcações adulteradas, em placas metálicas ferrosas, pela técnica de correntes parasitas (CP) /magnetografia (M) /visualização magneto-óptica (VMO) em comparação com o método destrutivo de restauração por ataque químico, utilizado pelos peritos criminais. Para tal, foram analisadas três amostras extraídas da sessão dianteira da estrutura do chassi de um veículo, as quais foram identificadas com caracteres impressos por indentações. Após a remoção das marcações por desgaste, as amostras foram analisadas pela inspeção CP/M/VMO e por ataque químico. Segundo os resultados obtidos, foi possível recuperar todas as identificações por ambos os métodos, além de compara-los segundo suas performances. Por conseguinte, a técnica não destrutiva demonstrou satisfatório desempenho na recuperação de marcações adulteradas.

Palavras-chave: Recuperação; Marcações Adulteradas; Visualização MagnetoÓptica; Ataque Químico.

\section{RESTORATION OF OBLITERATED MARKS ON METALLIC SAMPLES BY MAGNETO-OPTICAL IMAGING NON-DESTRUCTIVE METHOD}

\section{Abstract}

This work was carried out to test the efficiency of a magneto-optic imaging (MOI) ND method in the restoration of obliterated marks in metallic samples. This technology was compared to the destructive revelation method of chemical etching, which is the technique used by the criminal investigators. For that, three samples, extracted from a vehicle chassis structure, identified with codes designed by indentation marks, were analyzed by the previous discussed methods. The results showed that the original marks were recovered by both techniques. Moreover, the non-destructive method showed a promising performance in the recovery of obliterated marks.

Keywords: Recovery; Obliterated Marks; Magneto-Optic Imaging; Chemical Etching

1 Engenharia de Materiais, Engenheira, Aluna de Mestrado, Departamento de Engenharia Metalúrgica e de Materiais, Laboratório de Ensaios Não Destrutivos Corrosão e Soldagem, COPPE/UFRJ, Rio de Janeiro, Rio de Janeiro, Brasil.

2 Lic. Física, D.Sc., Pesquisadora, Laboratório de Ensaios Não Destrutivos Corrosão e Soldagem, COPPE/UFRJ, Rio de Janeiro, Rio de Janeiro, Brasil.

3 Engenharia Metalúrgica, D. Sc., Professora Adjunta, Departamento de Engenharia Metalúrgica e de Materiais, COPPE/UFRJ, Rio de Janeiro, Rio de Janeiro, Brasil.

4 Física, D. Sc., Professora Adjunta, Departamento de Engenharia Metalúrgica e de Materiais, Laboratório de Ensaios Não Destrutivos Corrosão e Soldagem, COPPE/UFRJ, Rio de Janeiro, Rio de Janeiro, Brasil.. 


\section{INTRODUÇÃO}

Após a implementação da ISO 3779, todo código de identificação veicular deve ser padronizado e impresso em áreas específicas de cada automóvel. Esse código tem como objetivo o rastreamento do veículo, ou peças deste, em qualquer transação. Entretanto, organizações criminosas no intuito de impossibilitar o rastreamento ou de executar uma venda ilícita, removem ou clonam o número de identificação veicular (NIV). As técnicas comumente empregadas para esse fim incluem: limagem, esmerilhamento, perfuração, soldagem, recobrimento, re-estampagem, regravação, entre outras.

Existem, entretanto, métodos específicos, muitos ainda em desenvolvimento, capazes de detectar e/ou recuperar a marcação pré-adulteração. Tanto a detecção quanto a recuperação dependem: do material, no qual será realizada a impressão, a técnica de marcação e o método de adulteração empregado. No momento em que um símbolo é impresso na superfície metálica do chassi, os grãos, nas regiões adjacentes a cavidade criada, serão deformados, alterando assim o alinhamento da estrutura cristalina local. Essas regiões são denominadas zonas de deformação plástica ou zonas de tensão residual, as quais se estendem ao redor da cavidade e ao longo da espessura do material, sendo sua profundidade dependente das características microestruturais do mesmo e do método de impressão utilizado.

Em adição, é importante ressaltar, que essas zonas apresentam propriedades físicas e químicas diferentes das zonas não deformadas, devido à variação da estrutura cristalinas nessas regiões. Desta forma, mudanças como variação de resistividade elétrica, variação de comportamento magnético e de potencial químico tornam possível a detecção das zonas de tensão residual por técnicas específicas, inclusive após a remoção da cavidade. (1)

Fundamentados nessa teoria, diversos métodos de recuperação de marcações originais vêm sendo estudados ao longo dos anos (Tabela 1). Entretanto, a maioria das técnicas validadas, para aplicação na área forense, se baseia em métodos destrutivos e são dependentes da composição química e do tratamento térmico, pelo qual o material foi submetido, para sua execução.

Tabela 1. Técnicas de recuperações de marcações originais. (2-Adaptado)(3)

\begin{tabular}{cc}
\hline Técnica & Detalhes \\
\hline Ataque Químico & Validado \\
\hline Ataque Eletrolítico & Validado \\
\hline Cavitação por Ultrassom & Validado \\
\hline Polimento de Alívio & Validado \\
\hline Tratamento Térmico & Validado \\
\hline Partículas Magnéticas & Validado \\
\hline Transmissão por Raios-x & Em desenvolvimento \\
\hline Reflexão de Raios- $x$ & Em desenvolvimento \\
\hline Difração de Elétrons Retroespalhados & Em desenvolvimento \\
\hline Visualização Magneto-Óptica & Em desenvolvimento \\
\hline
\end{tabular}

No Brasil, os peritos criminais fazem uso dos métodos de revelação por ataque químico e ataque eletrolítico, por se tratarem de técnicas validadas, de baixo custo e bem estabelecidas na indústria metalúrgica. Todavia, esses apresentam a desvantagem de se basearem em análises destrutivas, as quais fazem uso de agentes químicos nocivos à saúde do operador e ao ambiente, além de exigir o 
conhecimento sobre a dependência dos reagentes e a composição química do material.

Internacionalmente, $\mathrm{o}$ ataque químico também é amplamente utilizado pelos laboratórios forenses. Porém, existem, na literatura, estudos sobre a aplicação da inspeção por partículas magnéticas neste segmento. Entretanto, essa tecnologia se restringe apenas a materiais ferromagnéticos, tornando-se uma desvantagem na perícia de algumas motocicletas e carros de luxo, pois esses têm seus números de identificação impressos em superfícies metálicas paramagnéticas.

Baseados nesse cenário, cientistas forenses vêm estudando a aplicabilidade de métodos não destrutivos, já estabelecidos em certas aplicações de engenharia, além do desenvolvimento de novas tecnologias, que não comprometam a integridade do material analisado. Uma abordagem desenvolvida recentemente para detecção de adulterações é o sistema produzido pela empresa Letã Regula utilizado em diversos laboratórios forenses internacionais (4). Esse se baseia na evolução do ensaio de correntes parasitas convencional, no qual é possível obter a imagem dos defeitos por gravação magnética e conversão magneto-óptica.

Deste modo, o presente trabalho teve como objetivo analisar a aplicabilidade da técnica não destrutiva denominada como inspeção por correntes parasitas / magnetogramas / visualização magneto-óptica (CP/M/VMO), na revelação de marcações originais em comparação com a técnica destrutiva de recuperação por ataque químico.

\section{MATERIAIS E MÉTODOS}

\subsection{Calibrações do Equipamento}

O equipamento de inspeção não destrutiva de correntes parasitas (CP) / Magnetografia (M) / Visualização Magneto Óptica (VMO) foi inicialmente testado com o auxílio de amostras metálicas padrão com diferentes adulterações e de características ferromagnética e paramagnética (Figura 1). Estas apresentam caracteres com uma altura média de $9 \mathrm{~mm}$ e 200 a $300 \mu \mathrm{m}$ de profundidade.

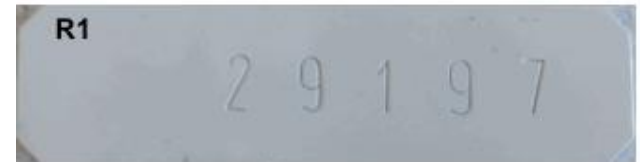

(a)

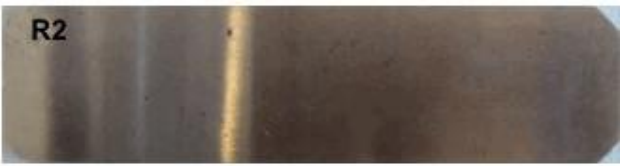

(b)

Figura 1. Amostras padrão: (a) R1, chapa paramagnética com adulteração simples; (b) R2, chapa ferromagnética com adulteração por remoção.

\subsection{Descrição das Amostras}

Neste estudo foram analisadas três amostras metálicas ferromagnéticas extraídas da seção dianteira da estrutura do chassi de um automóvel, categorizadas como: C1, C2 e C3.

Cada amostra foi identificada com um código específico, impressos por indentações Rockwell $C$ com uma carga de $62,5 \mathrm{~kg}$ (na tentativa de reproduzir a técnica de marcação por pontos ou micropercussão.

As informações quanto à dimensão das chapas, bem como altura e profundidade dos caracteres estão descritas na (Tabela 2). A altura dos caracteres e a 
profundidade média foram selecionadas de acordo com a norma ABNT e a resolução 659/85. $(5,6)$

A profundidade média de indentação foi medida com o auxílio de um perfilômetro Bruker, modelo Dektak XT.

Tabela 2. Descrição das amostras.

\begin{tabular}{ccccc}
\hline Amostras & Dimensões & Código & $\begin{array}{c}\text { Altura do } \\
\text { Caractere }\end{array}$ & $\begin{array}{c}\text { Profundidade } \\
\text { Média de } \\
\text { Indentação }\end{array}$ \\
\hline C1 & $\begin{array}{c}55 \mathrm{~mm} \times 30 \mathrm{~mm} \times \\
1,99 \mathrm{~mm}\end{array}$ & $\mathrm{CH} \Lambda 1$ & $7 \mathrm{~mm}$ & $230 \mu \mathrm{m}$ \\
\hline C2 & $\begin{array}{c}40 \mathrm{~mm} \times 35 \mathrm{~mm} \times \\
1,52 \mathrm{~mm}\end{array}$ & $\mathrm{LF}$ & $7 \mathrm{~mm}$ & $210 \mu \mathrm{m}$ \\
\hline C3 & $\begin{array}{c}33 \mathrm{~mm} \times 30 \mathrm{~mm} \times \\
1,55 \mathrm{~mm}\end{array}$ & $\mathrm{H}$ & $7 \mathrm{~mm}$ & $210 \mu \mathrm{m}$ \\
\hline
\end{tabular}

Em seguida, as placas foram adulteradas por remoção, ou seja, seus códigos de identificação foram removidos por desgaste, tornando-os imperceptíveis visualmente, na tentativa de simular casos em que a numeração é removida a fim de inviabilizar o rastreamento. Nessa etapa foram utilizadas lixas d'água de granulometria de $120 \mu \mathrm{m}$ (Figura 2).

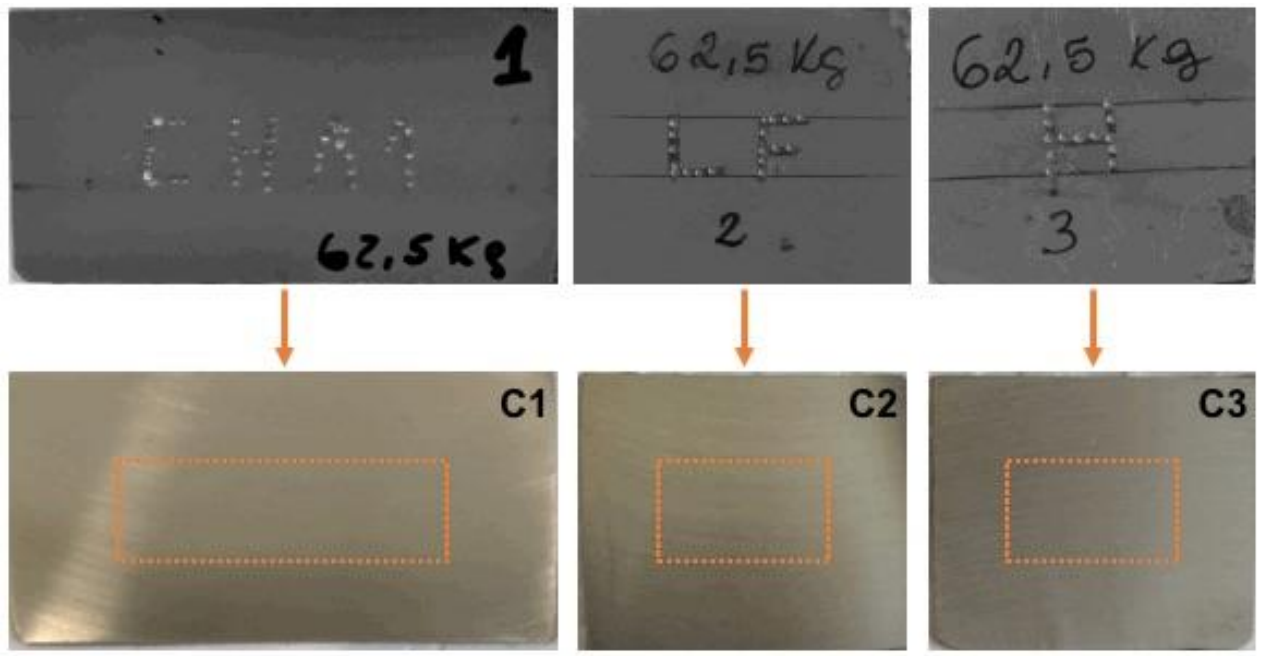

Figura 2. Amostras C1, C2 e C3, antes e após a remoção dos códigos de identificação.

$\mathrm{Na}$ adulteração, cada amostra sofreu uma perda de espessura diferente com o objetivo de avaliar o desempenho de ambas as técnicas de recuperação com relação a diferentes profundidades de zona de tensão residual.

Após a remoção, as chapas seguiram diretamente para etapa de recuperação não destrutiva por CP/M/VMO. Segundo a descrição do equipamento, este apresenta um limite máximo de não-planicidade superficial de $500 \mu \mathrm{m}$ e, a julgar pelas características superficiais de planicidade e rugosidade das amostras após a adulteração, não houve a necessidade de prepara-las metalograficamente para a análise em questão.

Entretanto, para a recuperação por ataque químico, foi necessário reduzir a rugosidade superficial ocasionada pela adulteração. Para isso, foi realizada uma preparação metalográfica, na qual foi utilizada a sequência de granulometrias de lixas d'água de: $220 \mu \mathrm{m}, 320 \mu \mathrm{m}, 400 \mu \mathrm{m}, 500 \mu \mathrm{m}, 600 \mu \mathrm{m}, 1200 \mu \mathrm{m}$ e $1500 \mu \mathrm{m}$, rotacionando a amostra por $90^{\circ}$ a cada mudança de lixa. 
A redução prévia da rugosidade superficial é apontada como de extrema importância por diversas literaturas para revelação macroscópica por ataque químico, pois as presenças de marcas grosseiras na superfície da amostra serão corroídas em taxas semelhantes às zonas de deformação, podendo assim interferir na interpretação do código, durante o ataque, devido à baixa variação de contraste entre o fundo e a marcação. (1,7-9)

As profundidades analisadas por cada método estão descritas na (Tabela 3). De acordo com as profundidades médias de indentação de cada amostra e a perda de espessura na remoção (Tabela 2 e Tabela 3), pode-se afirmar que as cavidades criadas, durante a impressão dos códigos, foram totalmente removidas. Desta forma, as técnicas foram utilizadas para detectar somente as zonas de deformação plásticas em diferentes profundidades.

Tabela 3. Perda de espessura após adulteração e após preparação metalográfica.

\begin{tabular}{cccccc}
\hline Amostras & $\begin{array}{c}\text { Espessura } \\
\text { Inicial }\end{array}$ & $\begin{array}{c}\text { Espessura } \\
\text { Após } \\
\text { Adulteração } \\
\text { (CP/M/VMO) }\end{array}$ & $\begin{array}{c}\text { Perda de } \\
\text { Espessura } \\
\text { Após } \\
\text { Adulteração }\end{array}$ & $\begin{array}{c}\text { Espessura } \\
\text { Após } \\
\text { Metalografia } \\
\text { (Ataque } \\
\text { Químico) }\end{array}$ & $\begin{array}{c}\text { Perda de } \\
\text { Espessura } \\
\text { Após } \\
\text { Metalografia }\end{array}$ \\
\hline C1 & $1,99 \mathrm{~mm}$ & $1,29 \mathrm{~mm}$ & $700 \mu \mathrm{m}$ & $1,15 \mathrm{~mm}$ & $840 \mu \mathrm{m}$ \\
\hline $\mathbf{C 2}$ & $1,52 \mathrm{~mm}$ & $1,20 \mathrm{~mm}$ & $320 \mu \mathrm{m}$ & $1,11 \mathrm{~mm}$ & $420 \mu \mathrm{m}$ \\
\hline C3 & $1,55 \mathrm{~mm}$ & $1,28 \mathrm{~mm}$ & $270 \mu \mathrm{m}$ & $1,17 \mathrm{~mm}$ & $380 \mu \mathrm{m}$ \\
\hline
\end{tabular}

\subsection{Recuperação por CP/M/VMO}

$\mathrm{Na}$ inspeção não destrutiva foram utilizados os equipamentos produzidos pela empresa Regula, sendo eles: Magnetografia por correntes parasitas de superfícies metálicas (modelo Regula 7505), o leitor magneto-óptico (modelo Regula 7515) e software NUCA para obtenção de imagem. $(10,11)$

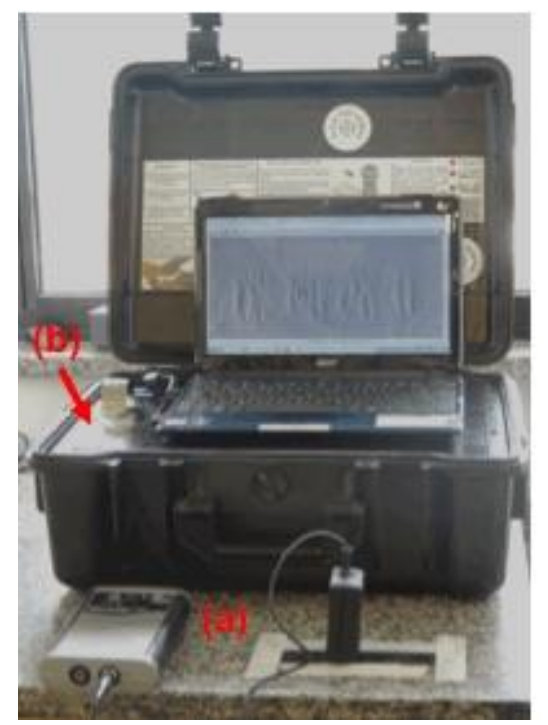

Figura 3. llustração do equipamento. (a) Indutor de correntes parasitas (Regula 7515); (b) Leitor Magneto-Óptico (Regula 7505).

A técnica não destrutiva por $\mathrm{CP} / \mathrm{M} / \mathrm{VMO}$ se baseia na suplementação do ensaio de correntes parasitas convencional, pela qual é possível obter a imagem de defeitos 
ou heterogeneidades superficiais ou subsuperficiais, do material analisado, através de gravação magnética e conversão magneto-óptica.

O ensaio é fundamentado na indução de correntes parasitas na superfície da amostra condutora, por um indutor, na utilização de uma fita magnética entre esse e a superfície da amostra, para a realização da gravação magnética e na conversão da informação magnética em imagem pelo leitor magneto-óptico (Figura 3).

Em outras palavras, quando o indutor varre a amostra e a fita magnética, correntes parasitas alternadas são induzidas na superfície do material condutor. Essas correntes alternadas geram um campo magnético secundário alternado atrelado a elas. Na presença de um defeito ou heterogeneidade na superfície, há a interação entre as correntes e as bordas dos defeitos, causando uma variação de circulação dessas e, consequentemente, uma mudança de polarização e intensidade do campo magnético secundário. A fita magnética, por ser composta de partículas magnéticas sensíveis a qualquer variação de polarização de campo magnético e capazes de armazenar a intensidade do mesmo por um longo período de tempo, registra a variação do campo magnético secundário nas bordas dos defeitos.

Realizado o decalque magnético, a fita é levada para o leitor magneto-óptico, onde será incidida uma onda eletromagnética linearmente polarizada sobre ela. Devido ao fenômeno de Rotação de Faraday, a luz polarizada, ao entrar em contato com o meio magnético, sofrerá uma rotação do seu plano de polarização de um ângulo proporcional a intensidade do campo magnético armazenado pela fita. Essa variação será posteriormente transformada em uma distribuição espacial de brilho e contraste criando assim uma imagem magneto-óptica do defeito. (12)

Com base neste fato, no experimento em questão foi adotado o seguinte procedimento, (10):

i. Preparação da Fita Magnética: Remoção de qualquer dado previamente armazenado com o auxílio de um imã permanente;

ii. Fixação da fita sobre a amostra com auxílio de fitas adesivas;

iii. Realização do Magnetograma: Varredura da superfície e da fita magnética com o indutor de correntes parasitas;

iv. Leitura da fita magnética pelo leitor magneto-óptico;

v. Processamento da imagem e análise do defeito: Correção de distorções do sistema óptico e junção panorâmica de imagens magneto-ópticas obtidas da magnetografia pelo software NUCA.

O tempo médio de análise para cada amostra contabilizando a preparação dos equipamentos, início do ensaio e obtenção de imagem foi cerca de 10 minutos.

\subsection{Recuperação por Ataque Químico}

Após a recuperação não destrutiva e preparação metalográfica as amostras seguiram para a etapa de recuperação por ataque químico.

Para a revelação das três chapas foi utilizado o reagente de Fry's de composição: $25 \mathrm{ml}$ de água $\left(\mathrm{H}_{2} \mathrm{O}\right), 30 \mathrm{ml}$ de ácido clorídrico $(\mathrm{HCl})$ e $22,5 \mathrm{~g}$ de cloreto de cobre dois $\left(\mathrm{CuCl}_{2}\right)$ recomendado para revelação macroscópica em aços carbono pela literatura. (2).

O procedimento adotado nesse ensaio se resumiu a: imersão das placas no ácido, remoção do excesso deste, secagem da amostra com uma toalha de papel e, por fim, registro dos resultados. Os tempos de análise de cada amostra estão descritos na (Tabela 4). 
Tabela 4. Tempo do ensaio por ataque químico de cada amostra.

\begin{tabular}{cc}
\hline Amostras & $\begin{array}{c}\text { Tempo de } \\
\text { Ataque }\end{array}$ \\
\hline C1 & 19 minutos \\
\hline C2 & 20 minutos \\
\hline C3 & 27 minutos \\
\hline
\end{tabular}

A aquisição de imagem das revelações foi realizada através de uma câmera fotográfica Nikon CoolPix P510.

\section{RESULTADOS E DISCUSSÃO \\ 3.1 Magnetografia}

$\mathrm{Na}$ calibração, o equipamento de mostrou apto a detectar adulterações e revelar as marcações originais em amostras ferromagnéticas e paramagnéticas com diferentes tipos de adulterações (Figura 4).
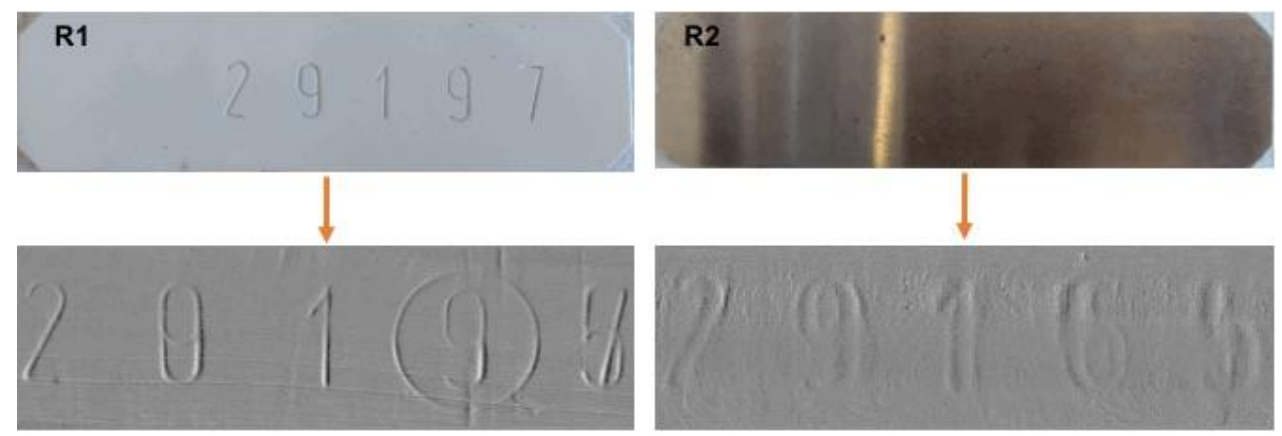

Figura 4. Magnetografias das amostras padrão: (a) R1 - placa paramagnética com adulteração simples e R2 - placa ferromagnética com adulteração por remoção; (b) Magnetografias obtidas das amostras R1 e R2.

Nas magnetografias das amostras C1, C2 e C3 foi possível identificar as marcações originais de todas as chapas após a adulteração (Figura 5).

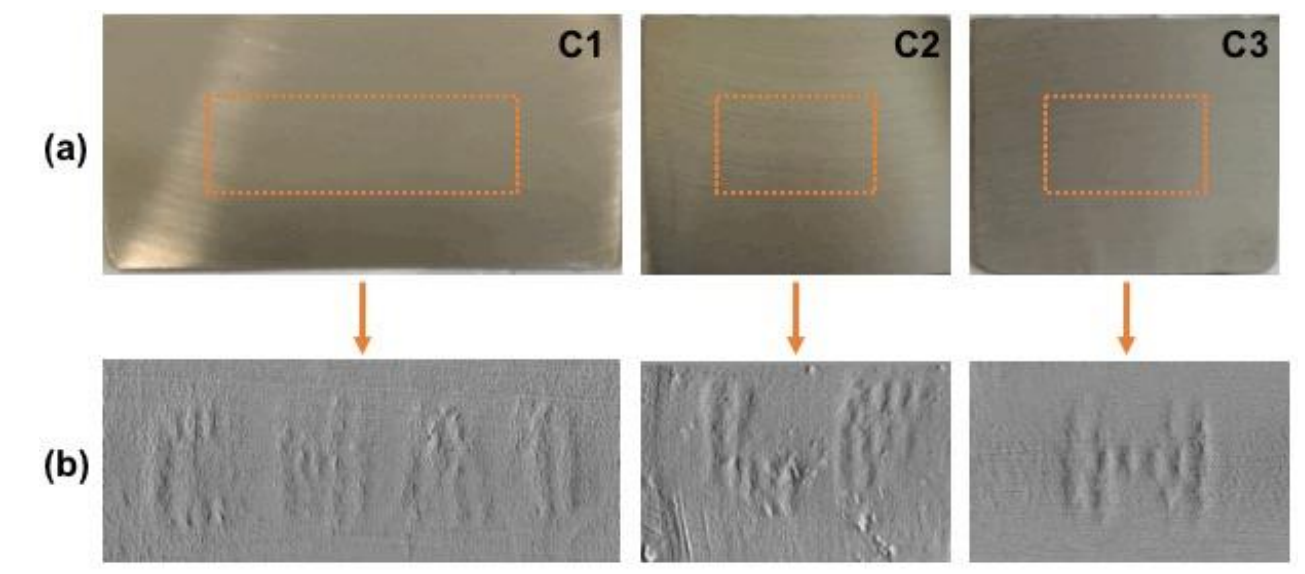

Figura 5. Magnetografia das amostras C's. Detecção das zonas de tensão residuais referentes a todos os caracteres: (a) Amostras com a marcação adulterada por remoção; (b) C1 - Revelação do código "CH$\Lambda 1$ ", $\mathbf{C 2}$ - Revelação do código "LF", C3 - Revelação do código "H".

A característica difusa dos caracteres nas amostras C's, pode ser explicada pela ausência dos orifícios ocasionados pelas impressões. Em casos em que a cavidade 
não fora totalmente removida, como na amostra padrão $\mathrm{R} 1$, a imagem magnetoóptica gerada apresenta maior definição, devido à lacuna de ar existente subsuperficialmente no material, o que resulta na variação de resistividade elétrica, variando intensamente a circulação das correntes parasitas e, consequentemente, 0 campo magnético secundário, armazenado pela fita magnética.

Em casos de total remoção do orifício gerado pela marcação, como nas amostras C's e na amostra padrão R2, a variação do campo magnético detectada pela fita é mais sutil, pois a mudança de propriedades elétricas e magnéticas entre a zona de deformação plástica e as zonas não deformada não são tão intensas quanto no caso da presença de defeitos internos, culminando em uma variação suave do campo magnético secundário.

\subsection{Ataque Químico}

$\mathrm{Na}$ Revelação por ataque químico das amostras C's foi possível detectar as marcações originais. Entretanto, foi necessária a realização de um polimento de alívio, após o ataque químico, para melhorar o contraste entre os códigos revelados e o fundo das chapas.

Após a adulteração (Figura 6a), os caracteres recuperados apresentaram baixa nitidez, dificultando a identificação, além disso, a rápida oxidação das amostras, após o ataque químico, tornou ainda mais desafiador o registro das imagens (Figura 6b). Na tentativa de melhora do contraste, foi aplicado o método de polimento de alívio com o auxilio de uma lixa de $1500 \mu \mathrm{m}$. Durante o polimento, partículas de óxido foram removidas em taxas diferentes, devido à variação da afinidade química entre o óxido e a regiões deformadas e não deformadas, tornando possível a melhora do contraste com a incidência de luz (Figura 6c).

(a)
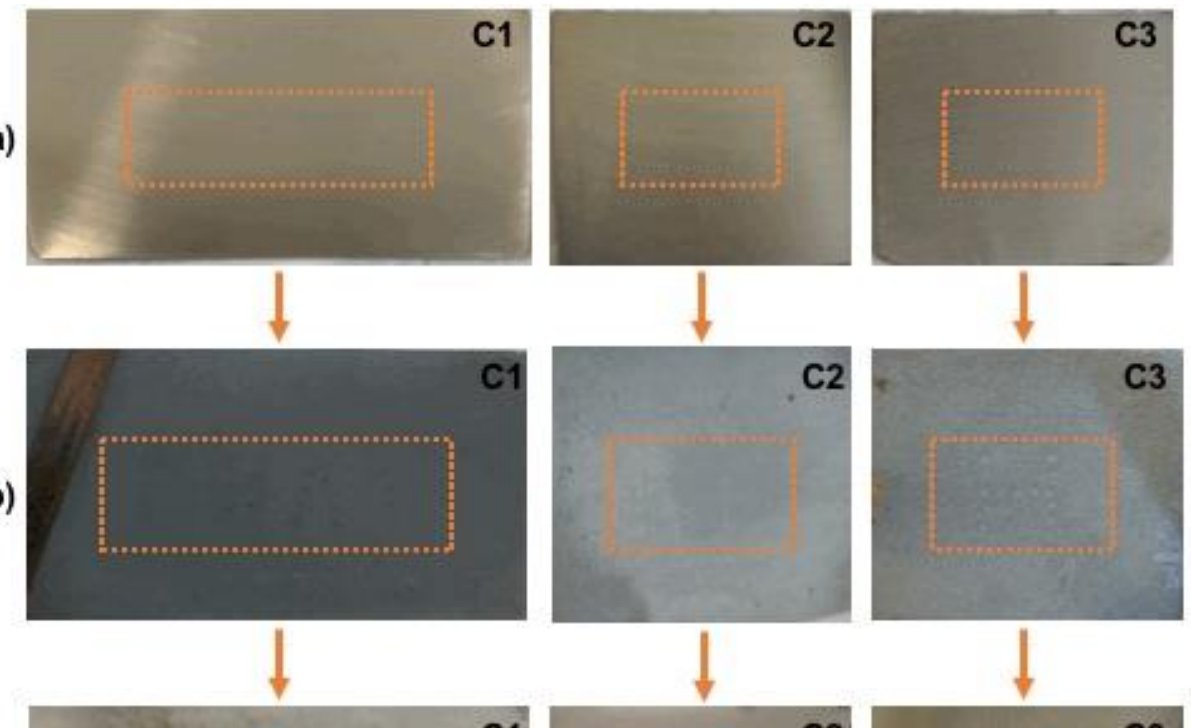

(c)
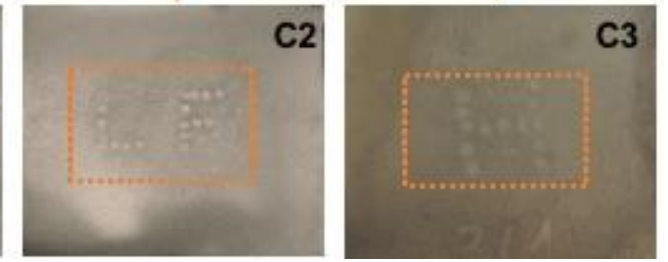

Figura 6. Revelação por ataque químico das amostras C's. (a) Amostras com a marcação adulterada por remoção; (b) Revelação por ataque químico; (c) Revelação após polimento de alívio. 
baixo contraste obtido no ataque químico, com o reagente em questão, pode ser explicado pela não compatibilidade química entre o reagente e o aço analisado, apesar de algumas literaturas apontarem o reagente de Fry's como ideal para revelações em aços, em geral. Devido ao fato da composição química dos metais, que compõem a estrutura do veículo, ser considerada segredo industrial pelas montadoras, a escolha do reagente ideal se torna cada vez mais desafiadora.

\subsection{Comparação Entre As Técnicas}

A técnica de recuperação por $\mathrm{CP} / \mathrm{M} / \mathrm{VMO}$ se mostrou superior à recuperação por ataque químico nos quesitos de tempo de análise, preservação de amostra, utilização de elementos nocivos à saúde e ao ambiente, dependência da composição química do material analisado, facilidade de operação e aquisição de imagem (Tabela 5).

Tabela 5. Comparação entre as técnicas.

\begin{tabular}{ccccccc}
\hline Amostras & $\begin{array}{c}\text { Tempo de } \\
\text { Análise }\end{array}$ & $\begin{array}{c}\text { Integridade } \\
\text { da Amostra }\end{array}$ & $\begin{array}{c}\text { Composição } \\
\text { Química do } \\
\text { Material } \\
\text { Analisado }\end{array}$ & $\begin{array}{c}\text { Utilização } \\
\text { de } \\
\text { Elementos } \\
\text { Nocivos }\end{array}$ & $\begin{array}{c}\text { Facilidade } \\
\text { de } \\
\text { Operação }\end{array}$ & $\begin{array}{c}\text { Aquisição } \\
\text { de } \\
\text { Imagem }\end{array}$ \\
\hline CP/M/VMO & $10 \mathrm{~min}$. & Preserva & Independente & Não faz uso & $\begin{array}{c}\text { Dispensa } \\
\text { Treinamento } \\
\text { Extensivos }\end{array}$ & $\begin{array}{c}\text { Fácil } \\
\text { Aquisição } \\
\text { Bom } \\
\text { Contraste }\end{array}$ \\
\hline $\begin{array}{c}\text { Ataque } \\
\text { Químico }\end{array}$ & $\approx 25 \mathrm{~min}$. & Não preserva & Dependente & Faz uso & $\begin{array}{c}\text { Necessidade } \\
\text { de } \\
\text { Experiência } \\
\text { do Operador }\end{array}$ & $\begin{array}{c}\text { Difícil } \\
\text { Aquisição } \\
\text { Contraste } \\
\text { Pobre. }\end{array}$ \\
\hline
\end{tabular}

\section{CONCLUSÃO}

Com base nos resultados obtidos, conclui-se que a técnica de recuperação, não destrutiva, por CP/M/VMO se mostrou capaz de recuperar as marcações originais de todas as amostras analisadas. Em adição, durante a calibração do equipamento, comprovou-se a competência da mesma em inspecionar peças de característica paramagnéticas, superando o método de inspeção por partículas magnéticas, em versatilidade.

A recuperação por ataque químico também se mostrou capaz de revelar as marcações originais, entretanto o reagente escolhido não se mostrou apto para revelação nas amostras estudadas. Este caso mostra a grande dependência dessa técnica quanto à composição química da peça analisada, sendo necessário o teste de outros reagentes químicos para melhores resultados.

Por fim, o método de CP/M/VMO apresentou satisfatório desempenho na inspeção de peças metálicas adulteradas por remoção e, de acordo com as análises realizadas até o presente momento, este têm se mostrado apto a ser incorporado ao conjunto de técnicas utilizadas na perícia forense.

\section{Agradecimentos}

A Capes, CNPq e a FAPERJ pelo apoio financeiro. A Regula, aos peritos da delegacia de roubos e furtos de automóveis (DRFA) e da delegacia de homicídios (DH) pelo auxílio técnico. 


\section{REFERÊNCIAS}

1. Kuppuswamy R. Metallographic Etching of Aluminium and Its Alloys for Restoration of Obliterated Marks in Forensic Science Practice and Investigations. In: Aluminium Alloys, theory and applications. 2010. p. 331-53.

2. Strauffer E, Bonfanti M. Restoration of Serial Numbers. In: Forensic Investigation of Stolen-Recovered and Other Crime Related Vehicles. 2006. p. 177-207.

3. Amato I. Scientists develop a technique to find serial numbers that have been filed off. Washington Post. Washington; 2015;

4. Weimar B, Herrmann D. A simple magneto-optical method for the restoration of erased markings in metals. Forensic Sci Int. Elsevier Ireland Ltd; $2011 ; 207(1-3): 119-21$.

5. ABNT. Veículos Rodoviários - número de identificação de veículos (VIN) ABNT NBR 6066. In: Associação Brasileira de Normas Técnica. 2001.

6. CONTRAN. Dispõe sobre o número de identificação de veículos. Brasil; 1985.

7. Vander Voort GF. Metallography, principles and practice. New York: McGraw Hill Book Company; 1984.

8. Vander Voort GF. Applied metallography. New York: Nostrand Reinhold Company; 1986.

9. Kehl GL. The principles of metallographic laboratory practice. McGraw Hill Book Company; 1949.

10. Science Systems RF. Operating Manual: Eddy-current magnetographing device of metal surface Regula model 7515. 2010.

11. Forensics R. Models 7505M e 7515, Product Information [Internet]. 2010 [cited 2017 Feb 15]. Available from: https://www.regulaforensics.com

12. Agalidi Y, Kozhukhar P, Levyi S, Rogozhinsky Y, Shumsky I. Eddy current fields/magnetic recording/magneto-optic imaging NDI method. Taylor Fr. 2011;27(2):109-19. 